\title{
Reconstruction of Typhoon Structure Using 3-Dimensional Doppler Radar Radial Velocity Data with the Multigrid Analysis: A Case Study in an Idealized Simulation Context
}

\author{
Hongli Fu, ${ }^{1}$ Xinrong Wu, ${ }^{1}$ Wei Li, ${ }^{1}$ Yuanfu Xie, ${ }^{2}$ Guijun Han, ${ }^{1}$ and Shaoqing $\mathrm{Zhang}^{3}$ \\ ${ }^{1}$ Key Laboratory of State Oceanic Administration for Marine Environmental Information Technology, \\ National Marine Data and Information Service, State Oceanic Administration, Tianjin 300171, China \\ ${ }^{2}$ NOAA Earth System Research Laboratory, Boulder, CO 80305-3328, USA \\ ${ }^{3}$ NOAA Geophysical Fluid Dynamics Laboratory, Princeton University, Princeton, NJ 08540-6649, USA \\ Correspondence should be addressed to Wei Li; liwei_nmdis@163.com
}

Received 25 March 2016; Revised 2 June 2016; Accepted 6 June 2016

Academic Editor: Mario M. Miglietta

Copyright ( 92016 Hongli Fu et al. This is an open access article distributed under the Creative Commons Attribution License, which permits unrestricted use, distribution, and reproduction in any medium, provided the original work is properly cited.

\begin{abstract}
Extracting multiple-scale observational information is critical for accurately reconstructing the structure of mesoscale circulation systems such as typhoon. The Space and Time Mesoscale Analysis System (STMAS) with multigrid data assimilation developed in Earth System Research Laboratory (ESRL) in National Oceanic and Atmospheric Administration (NOAA) has addressed this issue. Previous studies have shown the capability of STMAS to retrieve multiscale information in 2-dimensional Doppler radar radial velocity observations. This study explores the application of 3-dimensional (3D) Doppler radar radial velocities with STMAS for reconstructing a 3D typhoon structure. As for the first step, here, we use an idealized simulation framework. A two-scale simulated "typhoon" field is constructed and referred to as "truth," from which randomly distributed conventional wind data and 3D Doppler radar radial wind data are generated. These data are used to reconstruct the synthetic 3D "typhoon" structure by the STMAS and the traditional 3D variational (3D-Var) analysis. The degree by which the "truth" 3D typhoon structure is recovered is an assessment of the impact of the data type or analysis scheme being evaluated. We also examine the effects of weak constraint and strong constraint on STMAS analyses. Results show that while the STMAS is superior to the traditional 3D-Var for reconstructing the 3D typhoon structure, the strong constraint STMAS can produce better analyses on both horizontal and vertical velocities.
\end{abstract}

\section{Introduction}

Doppler radar has long been a valuable observational tool in meteorology. Three-dimensional (3D) Doppler radar radial velocity data can provide an opportunity to estimate both horizontal and vertical velocities. Therefore, in recent years, Doppler radar data assimilation for short-term numerical weather forecasting or called nowcasting has become a focal point of research [1-4]. Lots of techniques have been developed to retrieve wind field from Doppler radar radial velocity observations [2-24].

In Doppler radar radial velocity data assimilation used in the above literatures, in a three-dimensional variational (3D-Var) framework, a background error covariance matrix is always needed to determine the spatial spreading of observational information. It is well known that an analysis field at different locations may have different correlation scales [25], which are difficult to be estimated. Unfortunately, the traditional 3D-Var always employs an empirical and static background error covariance matrix and therefore usually can only correct single-scale wavelength error. However, the errors in short wavelength scales cannot be sufficiently corrected until the long waves are corrected $[25,26]$.

To minimize the errors of long and short waves in turn, a sequential 3D-Var approach has been proposed by Xie et al. [25, 26], implemented by either a recursive filter [27] or a multigrid technique [28] at Global Systems Division (GSD) of Earth System Research Laboratory (ESRL) in 
National Oceanic and Atmospheric Administration (NOAA) for a Federal Aviation Agency (FAA) project joined by the research team from the Lincoln Laboratory in Massachusetts Institute of Technology (MIT). Since this system also uses the temporal observation information, it is called a Space and Time Mesoscale Analysis System (STMAS, thereafter; see Xie et al. [26]). The STMAS has been applied to assimilating 2-dimensional (2D) Doppler radar radial velocity data to improve the wind field analyses [29].

Here, we study the analysis of 3D Doppler radar radial velocities using the STMAS to reconstruct the $3 \mathrm{D}$ wind structure. As for the first step, this study is performed in a twin experiment framework. In the next section, we first briefly review the theory of the multigrid 3D-Var data assimilation scheme in the STMAS. Some important aspects of the STMAS techniques such as smoothing, constraint, and Doppler radar radial wind operators used in the cost function of the STMAS multigrid 3D-Var are described. Section 3 first introduces the twin experiment framework for 3D Doppler radar radial wind data assimilation with the STMAS and then gives the evaluation by comparing it to the traditional 3DVar. Section 3 also examines the performance of the STMAS in weak and strong constraints for 3D Doppler radar radial velocity analysis. Conclusions and discussions are given in Section 4.

\section{Smoothing, Constraint, and Radar Radial Wind Operators in STMAS}

In this study, the STMAS implemented by the multigrid 3DVar is applied to the analysis of 3D Doppler radar radial velocities. This method can extract long and short wavelength information in turn efficiently from observations and provide objective and accurate analysis. The basic idea of this multigrid implementation can be referred to Li et al. [28-30].

To assimilate 3D Doppler radar radial velocities, with the control variables being $\mathbf{X}^{(n)}=\left(\mathbf{U}^{(n)^{T}}, \mathbf{V}^{(n)^{T}}\right)^{T}$, where $\mathbf{U}$ and $\mathbf{V}$ represent zonal and meridional components of wind vector, the cost functional for the $n$th level grid is

$$
\begin{aligned}
& J^{(n)}=J_{b}^{(n)}+J_{s}^{(n)}+J_{c}^{(n)}+J_{r}^{(n)} \quad(n=1,2,3, \ldots, N), \\
& J_{b}^{(n)}=\frac{1}{2} \mathbf{U}^{(n)^{T}} \mathbf{U}^{(n)}+\frac{1}{2} \mathbf{V}^{(n)^{T}} \mathbf{V}^{(n)}, \\
& J_{s}^{(n)}=\mathbf{U}^{(n)^{T}} \mathbf{S}_{\mathbf{U}} \mathbf{U}^{(n)}+\mathbf{V}^{(n)^{T}} \mathbf{S}_{\mathbf{V}} \mathbf{V}^{(n)},
\end{aligned}
$$

where the subscript $b$ denotes the background term and $s$ the smooth term, $c$ the conventional observation data term, and $r$ the radar radial wind observation data term. The smooth matrixes $S_{\mathbf{U}}$ and $\mathbf{S}_{\mathbf{V}}$ in the smooth term are derived from the Laplacian of control variables $\mathbf{U}$ and $\mathbf{V}$, respectively, at grid points. Let $\mathbf{W}$ represent the vertical component of wind vector. The details of the conventional observation data term and the 3D Doppler radar radial wind observation data term are as follows:

$$
\begin{aligned}
& J_{c}^{(n)}=\frac{1}{2}\left(\mathbf{H}^{(n)} \mathbf{U}^{(n)}-\mathbf{Y}_{\mathbf{U}}\right)^{T} \mathbf{O}_{\mathbf{U}}^{-1}\left(\mathbf{H}^{(n)} \mathbf{U}^{(n)}-\mathbf{Y}_{\mathbf{U}}\right) \\
& +\frac{1}{2}\left(\mathbf{H}^{(n)} \mathbf{V}^{(n)}-\mathbf{Y}_{\mathbf{V}}\right)^{T} \mathbf{O}_{\mathbf{V}}^{-1}\left(\mathbf{H}^{(n)} \mathbf{V}^{(n)}-\mathbf{Y}_{\mathbf{V}}\right) \\
& J_{r}^{(n)}=\frac{1}{2}\left(\boldsymbol{\Theta}_{\sin } \mathbf{H}^{(n)} \mathbf{U}^{(n)}+\boldsymbol{\Theta}_{\cos } \mathbf{H}^{(n)} \mathbf{V}^{(n)}+\mathbf{\Phi} \mathbf{H}^{(n)} \mathbf{W}^{(n)}\right. \\
& \left.\quad-\mathbf{Y}_{\mathbf{R}}\right)^{T} \mathbf{O}_{\mathbf{R}}^{(n)^{-1}}\left(\boldsymbol{\Theta}_{\sin } \mathbf{H}^{(n)} \mathbf{U}^{(n)}+\boldsymbol{\Theta}_{\cos } \mathbf{H}^{(n)} \mathbf{V}^{(n)}\right. \\
& \left.\quad+\boldsymbol{\Phi}^{(n)} \mathbf{W}^{(n)}-\mathbf{Y}_{\mathbf{R}}\right), \\
& \boldsymbol{\Theta}_{\sin }=\operatorname{diag}\left(\sin \theta_{1} \cos \varphi_{1}, \sin \theta_{2} \cos \varphi_{2}, \ldots\right. \\
& \left.\quad \sin \theta_{M} \cos \varphi_{M}\right), \\
& \boldsymbol{\Theta}_{\cos }=\operatorname{diag}\left(\cos \theta_{1} \cos \varphi_{1}, \cos \theta_{2} \cos \varphi_{2}, \ldots\right. \\
& \left.\quad \cos \theta_{M} \cos \varphi_{M}\right) \\
& \boldsymbol{\Phi}=\operatorname{diag}\left(\sin \varphi_{1}, \sin \varphi_{2}, \ldots, \sin \varphi_{M}\right)
\end{aligned}
$$

where $M$ is the amount of radar radial wind observations, $\theta$ is the azimuth angle of the radar beam relative to north with positive clockwise, and $\varphi$ is elevation angle of the radar beam. Of course, since radar scans at nonzero elevation angles, the fall speed of precipitation particles should be taken into account, and the algorithm of Sun and Crook [6] can be used to calculate terminal velocity. But for this study, we just neglect this terminal velocity, which does not lose its generality. The matrix $\mathbf{O}_{\mathbf{R}}^{(n)}$ is an error covariance matrix for radar radial wind observation; its superscript -1 stands for the reverse matrix, and its subscript $\mathbf{R}$ represents the radar radial wind observation.

During the procedure of sequential multiscale analyses, the operators $\mathbf{O}, \boldsymbol{\Theta}_{\text {sin }}, \boldsymbol{\Theta}_{\text {cos }}$, and $\boldsymbol{\Phi}$ remain the same when the full observation dataset is used through all multigrid levels; therefore, the superscript $(n)$ is omitted from these operators.

To make a strong constraint on these three components of wind vector, incompressible continuity equation $\partial \mathbf{U} / \partial x+$ $\partial \mathbf{V} / \partial y+\partial \mathbf{W} / \partial z=0$ is employed and discretized to calculate vertical velocity from the other two horizontal components. The discretized incompressible continuity equation is as follows:

$$
\begin{aligned}
& \mathbf{W}_{i, j, k}^{(n)}=\mathbf{W}_{i, j, k-1}^{(n)}+\frac{z_{k}^{(n)}-z_{k-1}^{(n)}}{2}\left(\frac{\mathbf{U}_{i+1, j, k-1}^{(n)}-\mathbf{U}_{i-1, j, k-1}^{(n)}}{x_{i+1}^{(n)}-x_{i-1}^{(n)}}\right. \\
& +\frac{\mathbf{V}_{i, j+1, k-1}^{(n)}-\mathbf{V}_{i, j-1, k-1}^{(n)}}{y_{j+1}^{(n)}-y_{j-1}^{(n)}}+\frac{\mathbf{U}_{i+1, j, k}^{(n)}-\mathbf{U}_{i-1, j, k}^{(n)}}{x_{i+1}^{(n)}-x_{i-1}^{(n)}} \\
& \left.+\frac{\mathbf{V}_{i, j+1, k}^{(n)}-\mathbf{V}_{i, j-1, k}^{(n)}}{y_{j+1}^{(n)}-y_{j-1}^{(n)}}\right) .
\end{aligned}
$$

The adjoint codes are recursively developed for $\mathbf{W}$ represented by $\mathbf{U}$ and $\mathbf{V}$. 


\section{Simulation Methodology}

3.1. Synthetic Typhoon Structure. The study domain covers a $500 \mathrm{~km} \times 500 \mathrm{~km}$ square region with $10 \mathrm{~km}$ thickness. The Doppler radar locates at the center $(250 \mathrm{~km}, 250 \mathrm{~km})$ of the study domain. A simulated typhoon field can be constructed by using the following function which consists of two subsection functions:

$$
F(L, D, r)= \begin{cases}1, & 0 \leq r \leq D, \\ \exp \left[-\frac{(r-D)^{4}}{L^{4}}\right], & r>D .\end{cases}
$$

This formula allows that the 2-order derivatives of this function exist. Let $r=\sqrt{\left(x-x_{c}\right)^{2}+\left(y-y_{c}\right)^{2}}$ and $G(x, y)=$ $A_{1} F\left[L_{1}, D_{1}, r(x, y)\right]+A_{2} F\left[L_{2}, D_{2}, r(x, y)\right]$, where $A_{1}=$ $-5 \mathrm{~km}^{2} \mathrm{~s}^{-1}, L_{1}=70 \mathrm{~km}, D_{1}=15 \mathrm{~km}, A_{2}=-1 \mathrm{~km}^{2} \mathrm{~s}^{-1}$, $L_{2}=20 \mathrm{~km}$, and $D_{2}=2.5 \mathrm{~km}$; then a stream function of a two-scale typhoon field can be constructed. The amplitude of one large-scale $\left(L_{1} / 2=35 \mathrm{~km}\right)$ is scaled by $A_{1}$ and the amplitude of one small-scale $\left(L_{2} / 2=10 \mathrm{~km}\right)$ is scaled by $A_{2}$. The locations of the maximum wind horizontal velocity of these two scales are $\sqrt[4]{0.75} L_{1}+D_{1} \approx 80 \mathrm{~km}$ for large-scale and $\sqrt[4]{0.75} L_{2}+D_{2} \approx 21 \mathrm{~km}$ for small-scale, respectively, from the typhoon center.

The typhoon center $\left(x_{c}, y_{c}\right)$ is set at $300 \mathrm{~km}$ and $150 \mathrm{~km}$. Then, the stream function and velocity potential function can be constructed as follows:

$$
\begin{aligned}
& \psi=G(x, y)\left(1-\exp \left(-\frac{z}{D_{3}}\right)\right), \\
& \chi=-\varepsilon G(x, y) \cos \left(\frac{z}{L_{3}} \pi\right),
\end{aligned}
$$

where $\varepsilon=0.5, L_{3}=10 \mathrm{~km}$, and $D_{3}=1 \mathrm{~km}$. The horizontal components can be expressed in terms of $\psi$ and $\chi$ :

$$
\begin{aligned}
& \mathbf{U}=\frac{\partial \chi}{\partial x}-\frac{\partial \psi}{\partial y} \\
& \mathbf{V}=\frac{\partial \chi}{\partial y}+\frac{\partial \psi}{\partial x}
\end{aligned}
$$

In this study, incompressibility is assumed. And the true vertical velocity field can be obtained by integrating the continuity equation $\partial \mathbf{U} / \partial x+\partial \mathbf{V} / \partial y+\partial \mathbf{W} / \partial z=0$. That is,

$$
\begin{aligned}
\mathbf{W} & =-\int_{0}^{z}\left(\frac{\partial \mathbf{U}}{\partial x}+\frac{\partial \mathbf{V}}{\partial y}\right) d z=-\int_{0}^{z}\left(\frac{\partial^{2} \chi}{\partial x^{2}}+\frac{\partial^{2} \chi}{\partial y^{2}}\right) d z \\
& =\frac{\varepsilon L_{3}}{\pi}\left(\frac{\partial^{2} G}{\partial x^{2}}+\frac{\partial^{2} G}{\partial y^{2}}\right) \sin \left(\frac{z}{L_{3}} \pi\right)
\end{aligned}
$$

where the bottom boundary condition is $\mathbf{W}(x, y, 0)=0$. The wind speed field in this simulated typhoon field contains two different scale information. The first one is about $35 \mathrm{~km}$ and the other is about $10 \mathrm{~km}$. The radial wind, $\mathbf{U}$ component, and $\mathbf{V}$ component pattern of middle level of this simulated typhoon field and a section wind field across the center of this typhoon are shown in Figure 1. This typhoon pattern is located at the southeast part of the study domain, so only the southeast square part is shown for the detailed structure. This simulated typhoon wind field is referred to as the "truth" typhoon field in this twin experiment.

3.2. Observations. Then, Doppler radar radial velocity data are generated from the "truth" typhoon field with one-degree azimuth angle increment and $2500 \mathrm{~m}$ gate spacing and 2degree elevation angle increment from 1 degree to 20 degrees by interpolating the "truth" velocity field to the radial velocity observations' points and using the equation $\mathbf{Y}_{\mathbf{R}}=\boldsymbol{\Theta}_{\sin } \mathbf{H U}+$ $\Theta_{\cos } \mathbf{H V}+\boldsymbol{\Phi} \mathbf{H W}$, and the number of radial velocity data is 114480. Spatially coarse (1000), moderate (10000), and dense (100000) random distributed conventional observations are also generated, respectively, from the "truth" typhoon field by interpolating the "truth" velocity field to the conventional observations' points.

3.3. Twin Experiments Setup. In the following, the above observational data are used to retrieve the simulated "typhoon" structure by the STMAS analysis method with weak or strong constraint and the traditional 3-dimensional variance (3D-Var) analysis with different correlation scales, respectively, and by comparing the analyzed results with the "truth," performances of different analysis methods are discussed.

The error variances of radial velocity observations and conventional observations can be determined by the measurement error of instruments. But, here for simplicity, the same error variance is set for each kind of data. However, because the amount of radial velocity data is much larger than that of conventional data, a scaling scheme is used to balance the weights of these two types of observations. Thus, the conventional observation can have the same weight as that of radial velocity observation, which may comprise these two types of observations to get to a reasonable wind analysis.

The limited memory BFGS (Broyden-Fletcher-GoldfarbShanno) method [31] to solve the bound constrained optimization problem [32] is used as the minimization method in this study.

3.4. STMAS Results. Three level grids are employed ranging from about $31.25 \mathrm{~km} \times 31.25 \mathrm{~km} \times 2.5 \mathrm{~km}(n=1$, and the number of grids is $17 \times 17 \times 5)$ to $7.8125 \mathrm{~km} \times 7.8125 \mathrm{~km} \times$ $0.625 \mathrm{~km}(n=3$, and the number of grids is $65 \times 65 \times 17)$ with grid ratio being 0.5 . The background is set to be zero for simplicity. The conventional data will be added gradually to investigate the impact of radar radial wind observations.

The STMAS analyses with the above 3D Doppler radar radial velocity data or conventional data which vary from coarse to dense are shown in Figures 2-4 and Table 1 gives the corresponding root mean square errors (RMSEs). Because the Doppler radar radial velocity data really provide some useful information of radial wind, the STMAS results by only using this type of data can make a good analysis on radial wind (see Figure 2(a)) and the two-scale information 


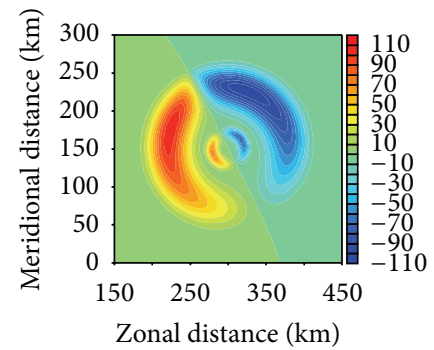

(a)

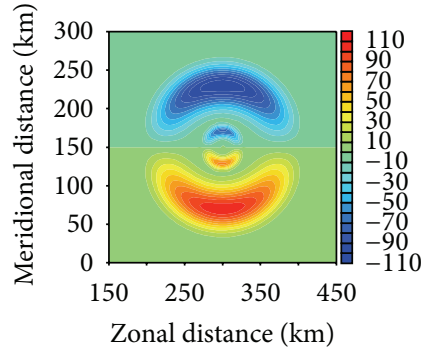

(b)

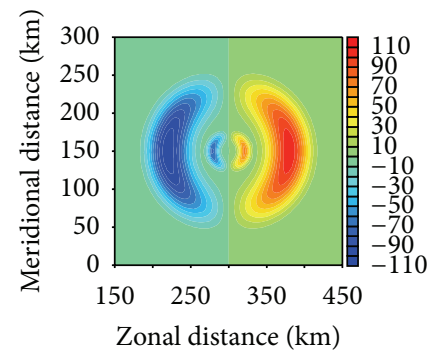

(c)

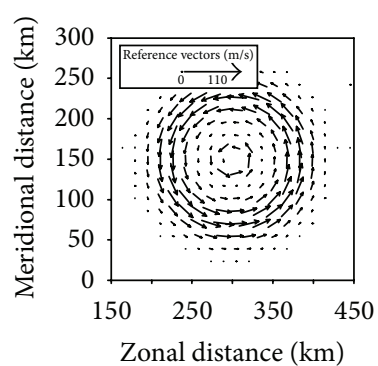

(d)

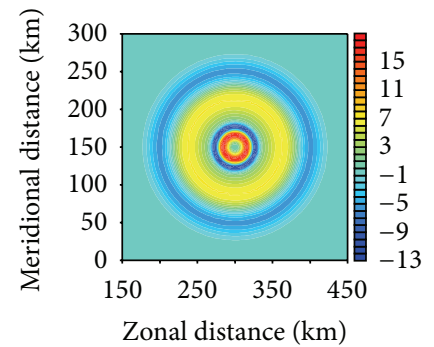

(e)

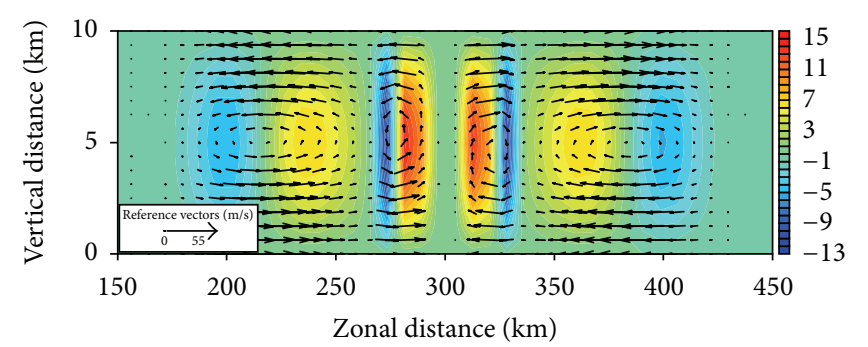

(f)

Figure 1: The synthetic "typhoon" field (serving as the "truth") at middle vertical level (5 km). (a) Radial velocity, (b) U component, (c) V component, (d) wind vector, (e) W component, and (f) W distribution at an $x-z$ section across the center of the synthetic "typhoon" with black arrows representing wind vector at this section (unit: $\mathrm{m} / \mathrm{s}$ ).

TABLE 1: Root mean square errors (RMSE) of $\mathbf{U}, \mathbf{V}$, and $\mathbf{W}$ component in different experiments. MG_RADAR represents the STMAS results by only assimilating radial wind data. MG_COARSE represents the STMAS results by assimilating only coarse conventional data. MG_RADAR_COARSE represents the STMAS results by assimilating both radial wind data and coarse conventional data. MG_MODERATE and MG_RADAR_MODERATE are similar to MG_COARSE and MG_RADAR_COARSE but with the conventional data substituted by moderate conventional data. MG_DENSE and MG_RADAR_DENSE are similar to MG_COARSE and MG_RADAR_COARSE but with the conventional data substituted by dense conventional data. MG_RADAR_DENSE_WEAK is similar to MG_RADAR_DENSE but with weak constraint. T50_RADAR_DENSE represents the results of traditional 3D-Var with correlation scales being $L_{x}=50 \mathrm{~km}, L_{y}=50 \mathrm{~km}$, and $L_{z}=5 \mathrm{~km}$ by assimilating both radial wind data and dense conventional data; T25_RADAR_DENSE is similar to T50_RADAR_DENSE but for $L_{x}=25 \mathrm{~km}, L_{y}=25 \mathrm{~km}$, and $L_{z}=2.5 \mathrm{~km}$; T12_RADAR_DENSE is similar to T50_RADAR_DENSE but for $L_{x}=12.5 \mathrm{~km}, L_{y}=12.5 \mathrm{~km}$, and $L_{z}=1.25 \mathrm{~km}$; T06_RADAR_DENSE is similar to T50_RADAR_DENSE but for $L_{x}=6.25 \mathrm{~km}, L_{y}=6.25 \mathrm{~km}$, and $L_{z}=0.625 \mathrm{~km}$.

\begin{tabular}{lccc}
\hline Experiment & RMSE of $\mathbf{U}(\mathrm{m} / \mathrm{s})$ & RMSE of V $(\mathrm{m} / \mathrm{s})$ & RMSE of W $(\mathrm{m} / \mathrm{s})$ \\
\hline MG_RADAR & 15.7032 & 14.5197 & 2.3663 \\
MG_COARSE & 9.2592 & 9.2195 & 1.4610 \\
MG_RADAR_COARSE & 7.9500 & 7.6029 & 1.3757 \\
MG_MODERATE & 3.2196 & 3.1966 & 0.8228 \\
MG_RADAR_MODERATE & 3.0649 & 2.6148 & 0.7930 \\
MG_DENSE & 1.0707 & 1.0849 & 0.5494 \\
MG_RADAR_DENSE & 1.0514 & 1.0135 & 0.5373 \\
MG_RADAR_DENSE_WEAK & 1.0747 & 1.0254 & 2.0840 \\
T50_RADAR_DENSE & 6.8944 & 7.1118 & 1.3832 \\
T25_RADAR_DENSE & 2.8425 & 2.7135 & 0.8172 \\
T12_RADAR_DENSE & 3.7725 & 3.6773 & 0.7787 \\
T06_RADAR_DENSE & 10.2998 & 10.2631 & 1.3669 \\
\hline
\end{tabular}

in radial wind has been captured by the STMAS. There is no information of tangential wind, and no statistical or empirical correlation information between $\mathbf{U}$ and $\mathbf{V}$ is used in this study. Therefore, only using radial velocity data, the STMAS analysis only can match the radial wind, and it cannot make a good analysis on $\mathbf{U}$ and $\mathbf{V}$. This leads to large $\mathbf{U}$ and $\mathbf{V}$
RMSEs $(15.7 \mathrm{~m} / \mathrm{s}$ and $14.5 \mathrm{~m} / \mathrm{s}$, resp., see Table 1$)$. With the 1000 coarse distributed conventional data only, the STMAS analysis only shows the large pattern of the typhoon wind (Figure 2(b)). With the combination of conventional data and radar radial wind data, the STMAS analysis significantly improves the typhoon structure (Figure 2(c)). The RMSE 

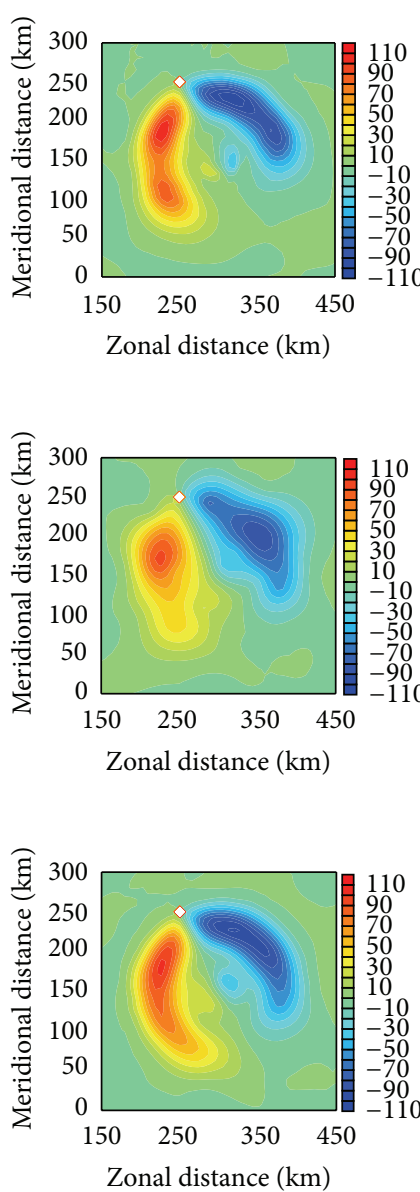

(c)

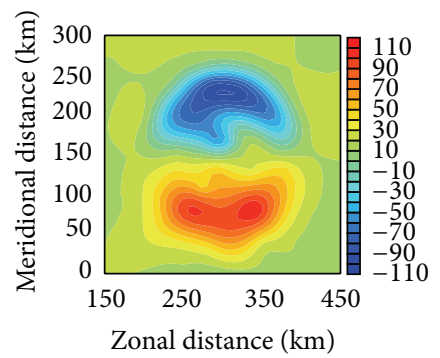

(b)
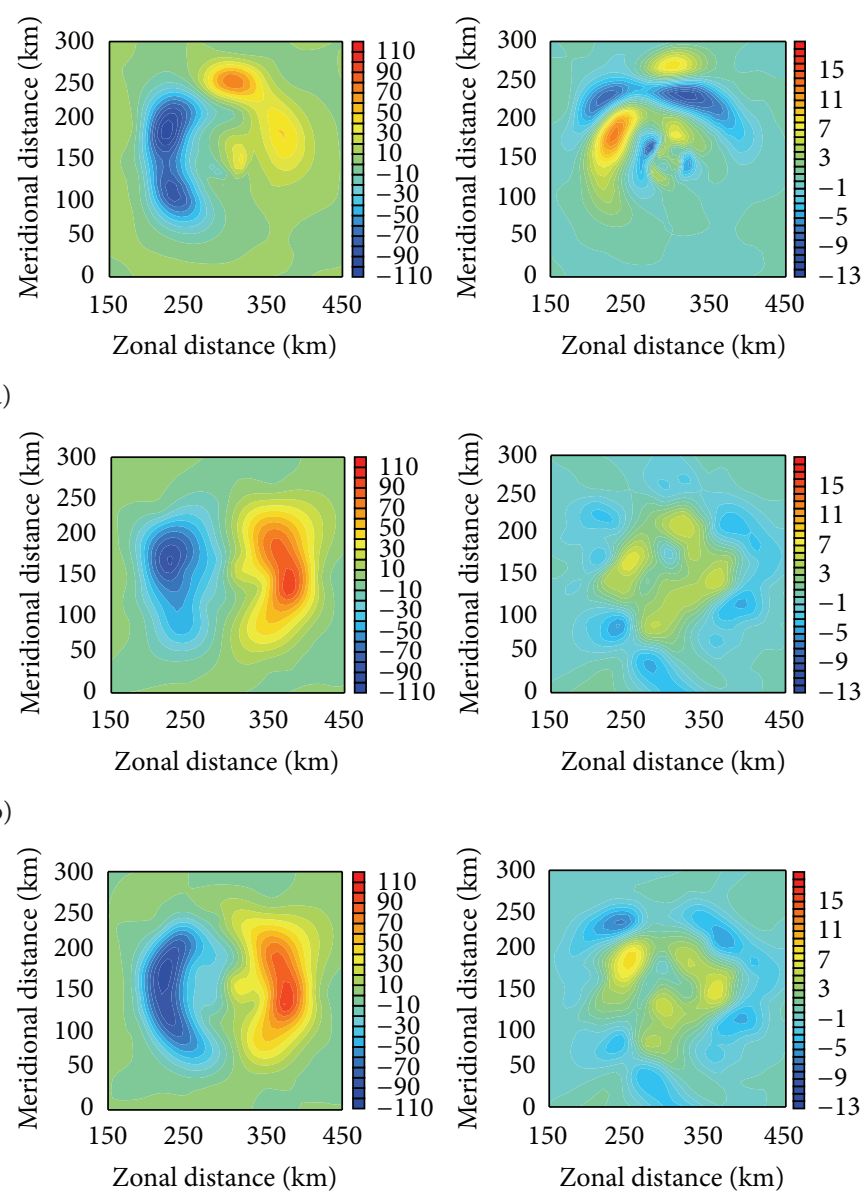

(a)
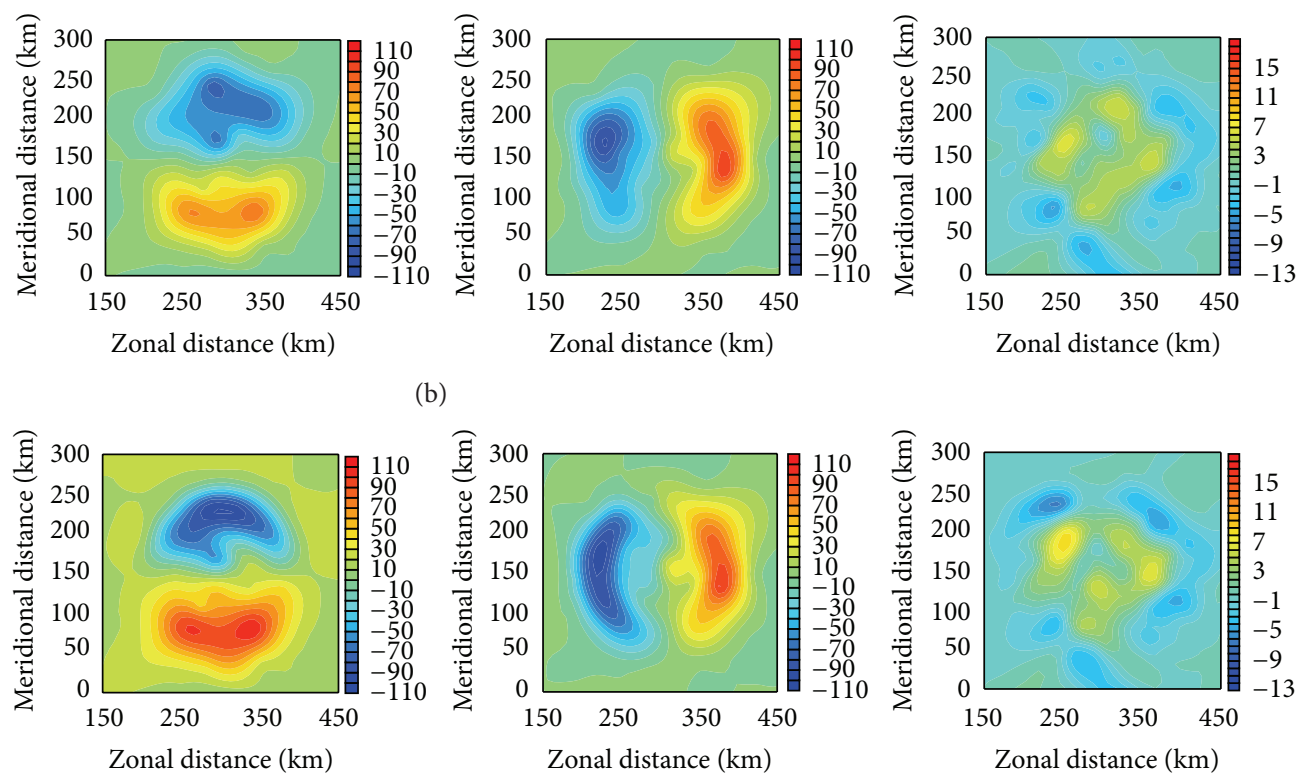

FIGURE 2: STMAS analyses at middle vertical level. From left to right, these columns represent radial wind, U component, V component, and W component, respectively. (a) is the results by only assimilating radial wind data. (b) is the results by assimilating only coarse conventional data. (c) is the results by assimilating both radial wind data and coarse conventional data (unit: $\mathrm{m} / \mathrm{s}$ ).

with the combined datasets is much smaller than the other one with an individual dataset (Table 1). Apparently, the radial velocity data provide additional information on radial direction to help the STMAS capture the detailed typhoon structure. If the conventional data change from the coarsest (totally 1000) to the densest (totally 100000), the detailed information on the typhoon is enhanced gradually (see Figures 2(b) and 2(c) corresponding to 1,000 conventional data and 1,000 conventional data combined with radar data, resp.; Figures 3(a) and 3(b) corresponding to 10,000 conventional data and 10,000 conventional data combined with radar data, resp.; Figures 4(a) and 4(b) corresponding to 100,000 conventional data and 100,000 conventional data combined with radar data, resp.). With the combination of radar radial velocity data and dense distributed conventional data, the STMAS can make almost perfect analysis for the typhoon structure (Figure 4(b)) and the RMSE is very small. And because dense distributed conventional data provide enough information, the RMSE improvements from the MG_DENSE to MG_RADAR_DENSE experiments are really negligible for the three wind components.
3.5. Strong Constraint versus Weak Constraint. To compare the performance of the STMAS with a strong constraint or weak constraint, the continuity equation is added as penalty term to make a weak constraint case (otherwise, the STMAS analysis is a strong constraint of the three components of wind vector). Then, the control variables become $\mathbf{X}^{(n)}=$ $\left(\mathbf{U}^{(n)^{T}}, \mathbf{V}^{(n)^{T}}, \mathbf{W}^{(n)^{T}}\right)^{T}$ :

$$
\begin{aligned}
J^{(n)}=J_{b}^{(n)}+J_{s}^{(n)}+J_{c}^{(n)}+J_{r}^{(n)}+J_{\text {constraint }}^{(n)} & \\
& (n=1,2,3, \ldots, N) .
\end{aligned}
$$

And background term is changed to

$$
J_{b}^{(n)}=\frac{1}{2} \mathbf{U}^{(n)^{T}} \mathbf{U}^{(n)}+\frac{1}{2} \mathbf{V}^{(n)^{T}} \mathbf{V}^{(n)}+\frac{1}{2} \mathbf{W}^{(n)^{T}} \mathbf{W}^{(n)}
$$

The smooth term is

$$
J_{s}^{(n)}=\mathbf{U}^{(n)^{T}} \mathbf{S}_{\mathbf{U}} \mathbf{U}^{(n)}+\mathbf{V}^{(n)^{T}} \mathbf{S}_{\mathbf{V}} \mathbf{V}^{(n)}+\mathbf{W}^{(n)^{T}} \mathbf{S}_{\mathbf{W}} \mathbf{W}^{(n)}
$$




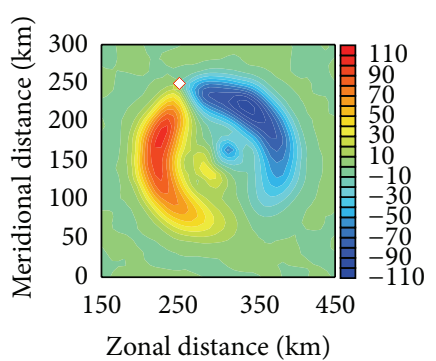

Zonal distance $(\mathrm{km})$

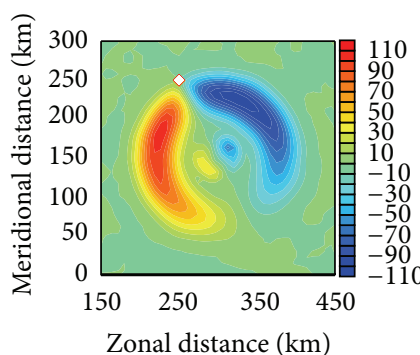

Zonal distance $(\mathrm{km})$
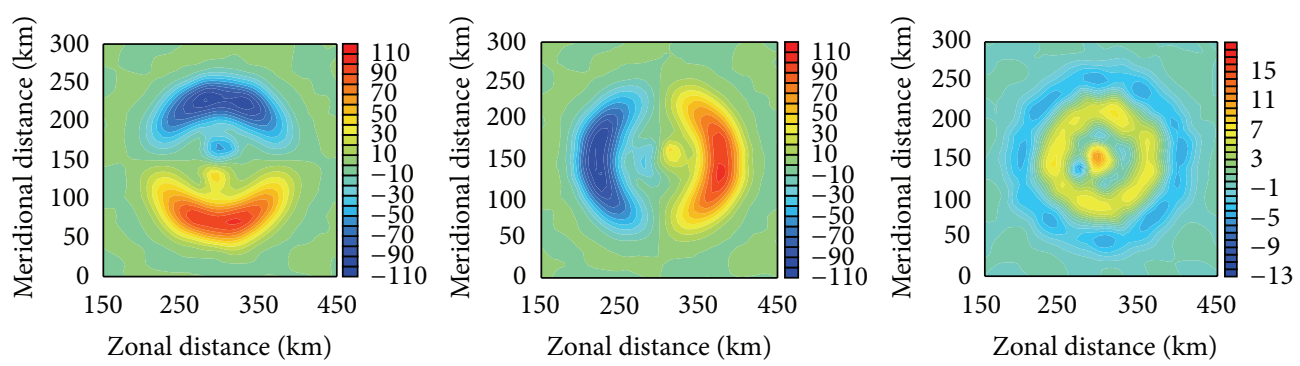

(a)

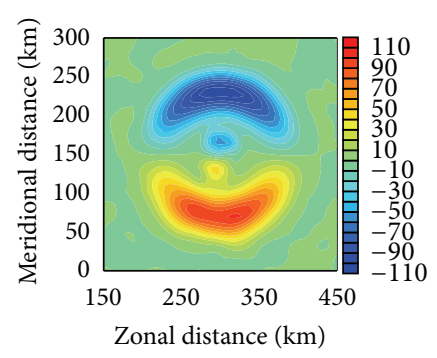

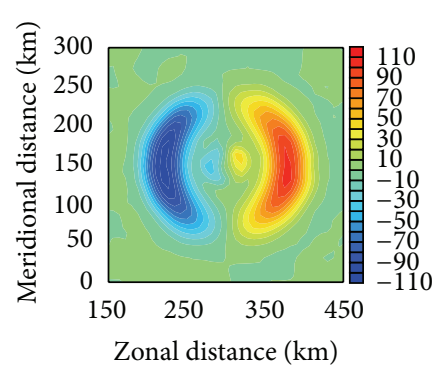

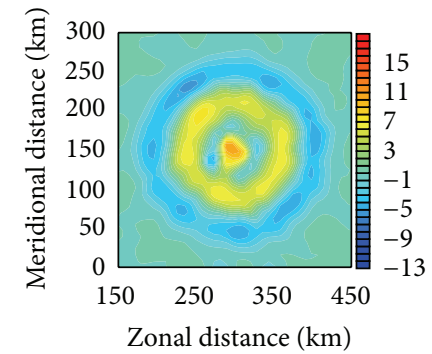

(b)

FIGURE 3: (a) and (b) are similar to Figures 2(b) and 2(c) but with the conventional data substituted by moderate conventional data (unit: $\mathrm{m} / \mathrm{s})$.

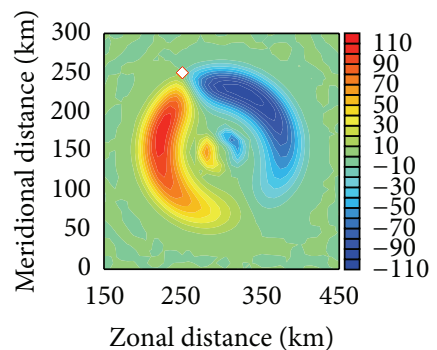

Zonal distance $(\mathrm{km})$

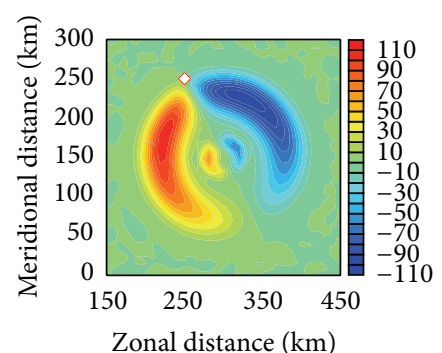

Zonal distance $(\mathrm{km})$

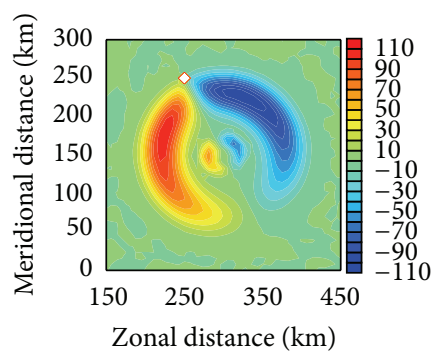

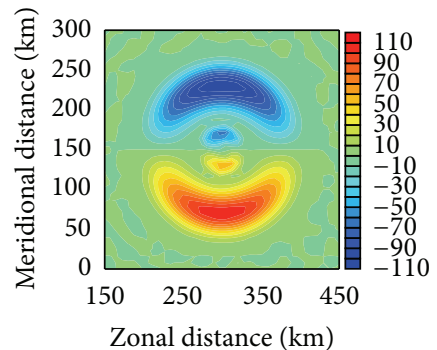

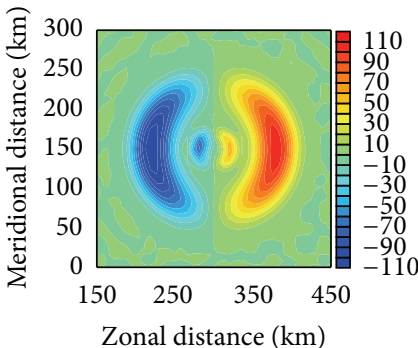

(a)

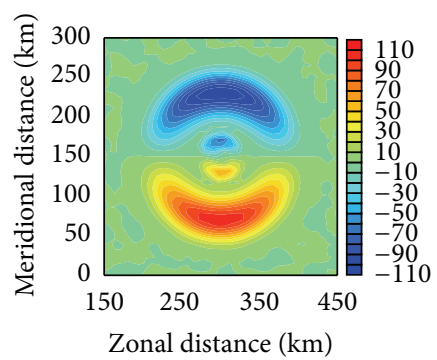

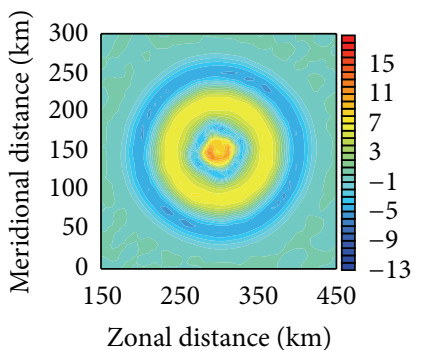

Zonal distance $(\mathrm{km})$

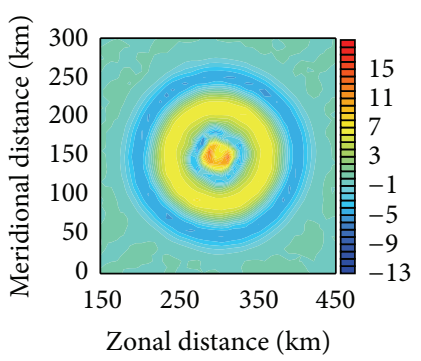

(b)

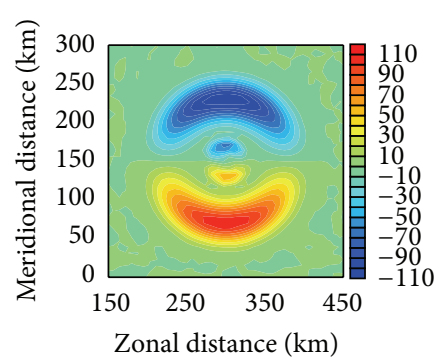

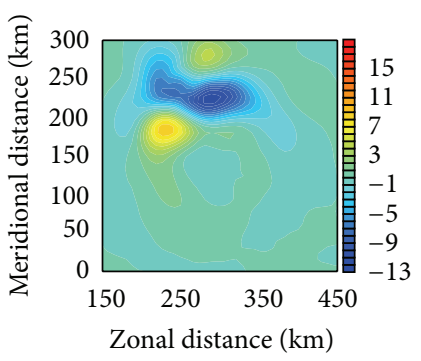

Zonal distance $(\mathrm{km})$

(c)

FIgURE 4: (a) and (b) are similar to Figures 2(b) and 2(c) but with the conventional data substituted by dense conventional data. (c) is similar to (b) but with weak constraint (unit: $\mathrm{m} / \mathrm{s}$ ). 
Here, $\mathbf{S}_{\mathbf{W}}$ is smooth matrix for $\mathbf{W}$ component of wind vector. The weak constraint term is

$$
J_{\text {constraint }}^{(n)}=\alpha \int\left(\frac{\partial \mathbf{U}^{(n)}}{\partial x}+\frac{\partial \mathbf{V}^{(n)}}{\partial y}+\frac{\partial \mathbf{W}^{(n)}}{\partial z}\right)^{2} d x d y d z
$$

where $\alpha$ is the penalty coefficient for weak constraint. The other terms, $J_{c}^{(n)}$ and $J_{r}^{(n)}$, are the same as (2) and (3), respectively.

Assimilating the whole simulated Doppler radar radial velocity data and dense conventional data, the STMAS analyses with continuity equation as strong constraint or weak constraint are shown in Figures 4(b) and 4(c), respectively. And the RMSE is shown in Table 1 . The coefficient of penalty term for weak constraint is set to 100 .

As shown in Figure 4(b), the strong constraint forces these three components of wind vector to exactly satisfy the continuity equation. Then, while the horizontal components can be analyzed very well, the vertical component is reconstructed very well too. The weak constraint (Figure 4(c)) can only constrain the horizontal and vertical components of wind vector to satisfy the continuity equation to some degree. Whereas the order of vertical velocities is much smaller than that of horizontal ones and no direct observation of vertical velocities is available, the minimization of cost function primarily focuses on the horizontal parts. This causes little improvement on the vertical velocities even if the horizontal components can be well analyzed. Therefore, the vertical component RMSE score of MG_RADAR_DENSE_WEAK experiment is worse than that of MG_RADAR_DENSE experiment. When a large penalty coefficient $\alpha$ is used, although vertical velocity analysis can be improved a little bit suffering a lower accuracy of horizontal velocity, the accuracy of the analysis vertical velocities is still not high.

3.6. STMAS versus Traditional 3D-Var. For a traditional 3DVar analysis, the cost function takes the form

$$
\begin{aligned}
J & =J_{b}+J_{c}+J_{r}, \\
J_{b} & =\frac{1}{2} \mathbf{U}^{T} \mathbf{B}_{\mathbf{U}}^{-1} \mathbf{U}+\frac{1}{2} \mathbf{V}^{T} \mathbf{B}_{\mathbf{V}}^{-1} \mathbf{V} .
\end{aligned}
$$

For simplicity, here, we assume $\mathbf{B}_{\mathbf{U}}=\mathbf{B}_{\mathbf{V}}=\mathbf{B}$, and no covariance between the two horizontal velocity components is considered. The background error covariance matrix $\mathbf{B}$ takes the following form [33]:

$$
\mathbf{B}_{i, j}=a_{h} \exp \left(-\frac{\Delta x_{i j}^{2}}{L_{x}^{2}}-\frac{\Delta y_{i j}^{2}}{L_{y}^{2}}-\frac{\Delta z_{i j}^{2}}{L_{z}^{2}}\right),
$$

where $L_{x}, L_{y}$, and $L_{z}$ are characteristic length scales which reflect the extent of spatial correlation; $x y$ and $z$ are coordinates, and $i$ and $j$ are indexes of grid point; and $a_{h}$ is the firstguess error variance. $J_{c}$ and $J_{r}$ are similar to formulas (2) and (3), respectively, but with an omitted superscript $(n)$. Strong constraint is also imposed on $\mathbf{U}, \mathbf{V}$, and $\mathbf{W}$ component by using discretized continuity equation. To make a comparison with STMAS, the dense conventional data and Doppler radar radial velocity data are all used. The RMSE is shown in Table 1.
Based on Gaussian distribution, the traditional 3D-Var using correlation scale usually constructs the background error covariance matrix by an empirical correlation scale. Therefore, the traditional 3D-Var with a certain correlation scale only can analyze this kind of scale information. However, the "truth" typhoon field in this study contains two different spatial scales wind speed information $(\sim 35 \mathrm{~km}$ and $\sim 10 \mathrm{~km}$ ). The traditional 3D-Var with $50 \mathrm{~km}$ horizontal correlation scale can only capture the main pattern of this typhoon field (i.e., the long wave information) but lose the small-scale information and produce a smooth analysis (Figure 5(a)). The traditional 3D-Var with $25 \mathrm{~km}$ or $12.5 \mathrm{~km}$ horizontal correlation scale can capture some detailed information of the long wave as well as major short wave information, but detailed short wave information cannot be well analyzed (Figures 5(b) and 5(c)). On the contrary, the traditional 3D-Var with $5 \mathrm{~km}$ correlation scale can analyze short wave features but incorrectly treats long wave information. This leads to an erroneous analysis (Figure 5(d)). The two scales of this true typhoon field are $35 \mathrm{~km}$ and $10 \mathrm{~km}$, respectively, and the large-scale $(35 \mathrm{~km})$ component covers most of study domain. In the experiment T25_RADAR_DENSE, the horizontal correlation scale is $25 \mathrm{~km}$ which is the closest to the large-scale $(35 \mathrm{~km})$ of the true typhoon field among all these traditional experiments; therefore, the horizontal wind velocity analysis is the best.

From the vertical velocity distribution as well as wind vector shown in Figure 6, we can further verify the above argument. Near the typhoon center, there are two branches of vertical circulations on each side: one is narrow and the other is broad, corresponding to four upwelling zones in this section. Using the dense conventional data and Doppler radar radial velocity data, the STMAS can analyze these sets of vertical circulations and corresponding upwelling zones (Figure 6(a)). However, the traditional 3D-Var with horizontal correlation scales being $50 \mathrm{~km}, 25 \mathrm{~km}$, or $12.5 \mathrm{~km}$ can only analyze two (Figure 6(b)) or three (Figures 6(c) and 6(d)) upwelling zones, since these kinds of correlation scales filter out the small-scale information and merge these upwelling zones. Although the traditional 3D-Var with $6.25 \mathrm{~km}$ horizontal correlation scale can distinguish the middle two upwelling zones, it incorrectly treats the large-scale information (Figure 6(e)).

\section{Conclusions and Discussions}

Within an idealized simulation framework, the role of 3D Doppler radar radial velocity data for reconstructing 3D typhoon structures has been examined using the Space and Time Mesoscale Analysis System (STMAS). A two-scale simulated "typhoon" field is constructed and referred to as "truth," from which randomly distributed conventional wind data and 3D Doppler radar radial wind data are generated. These data are used to reconstruct the synthetic 3D "typhoon" structure by the STMAS or the traditional 3D variational (3DVar) analysis. The degree by which the "truth" 3D typhoon structure is recovered is an assessment of the impact of data type or analysis scheme being evaluated. The effects of 

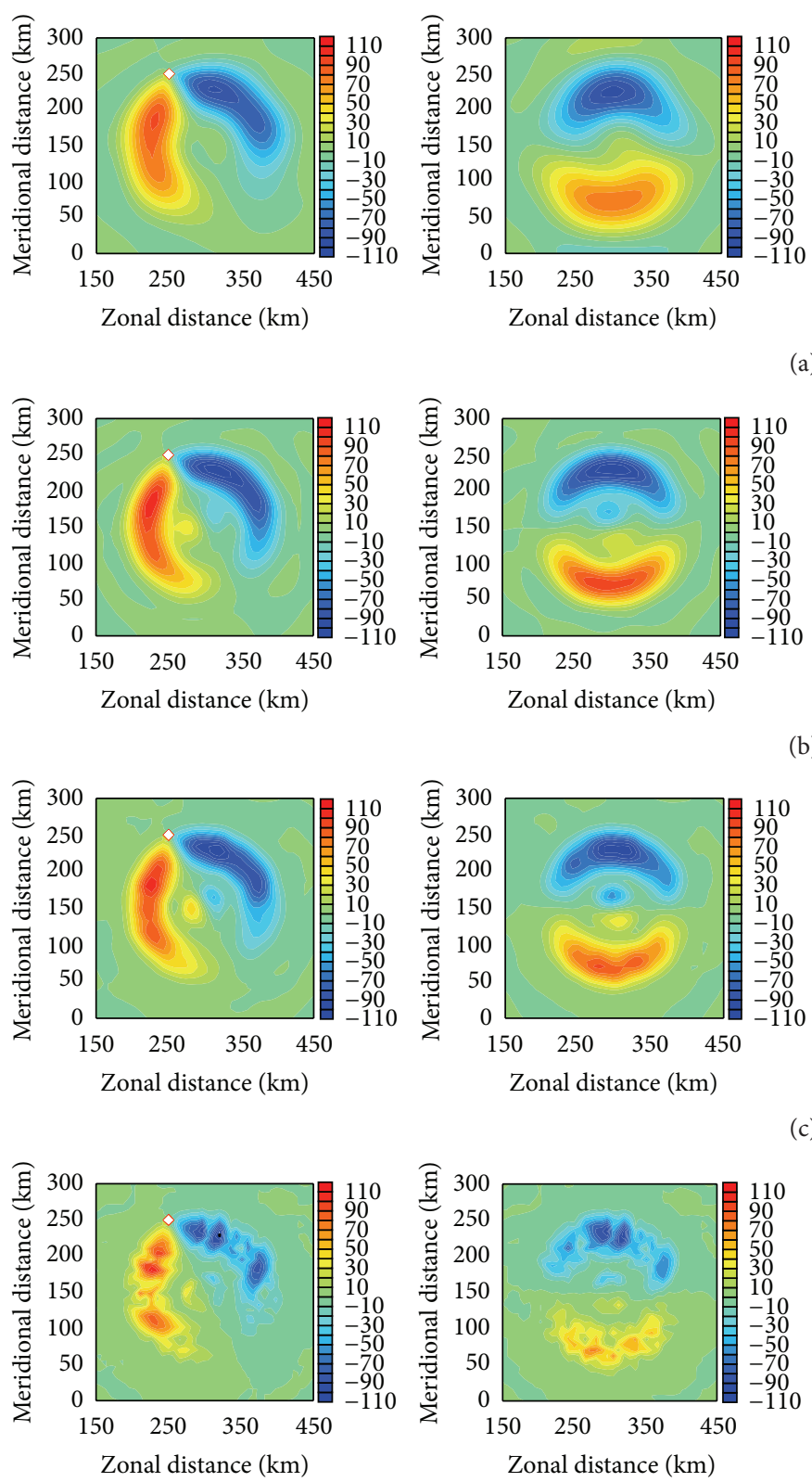

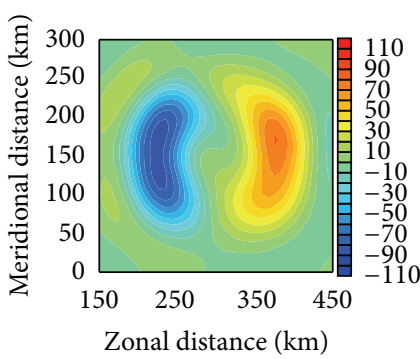

(a)

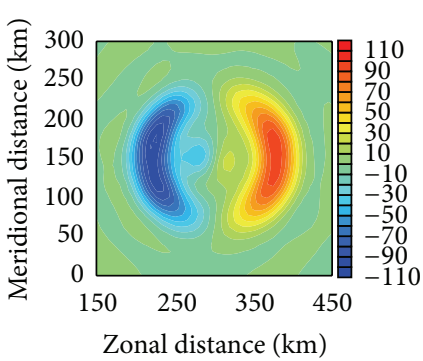

(b)

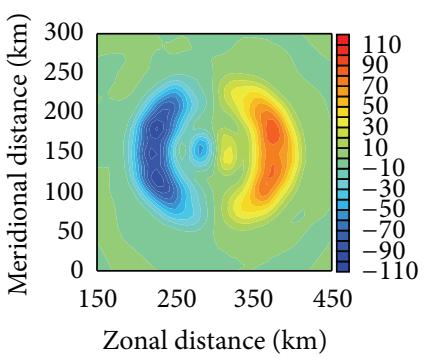

(c)
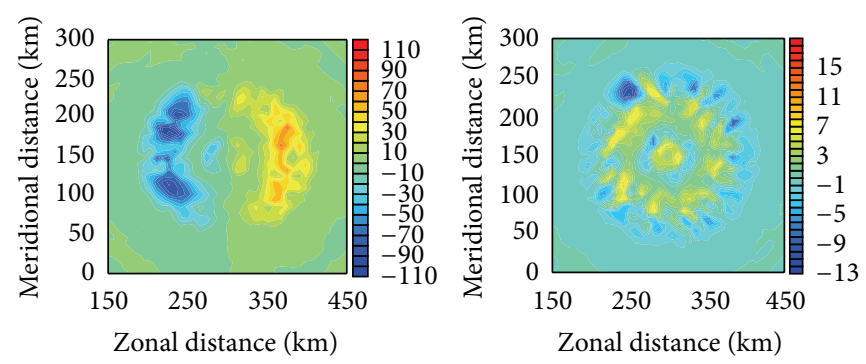
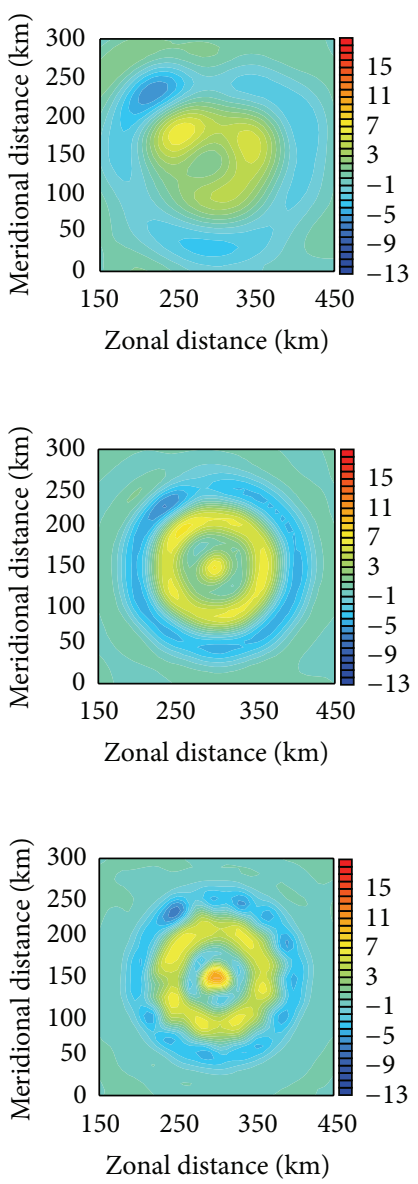

(d)

Figure 5: Similar to Figure 4(b) but with the results of traditional 3D-Var with correlation scales being $L_{x}=50 \mathrm{~km}, L_{y}=50 \mathrm{~km}$, and $L_{z}=5 \mathrm{~km} \mathrm{(a);} L_{x}=25 \mathrm{~km}, L_{y}=25 \mathrm{~km}$, and $L_{z}=2.5 \mathrm{~km}(\mathrm{~b}) ; L_{x}=12.5 \mathrm{~km}, L_{y}=12.5 \mathrm{~km}$, and $L_{z}=1.25 \mathrm{~km}(\mathrm{c}) ;$ and $L_{x}=6.25 \mathrm{~km}$, $L_{y}=6.25 \mathrm{~km}$, and $L_{z}=0.625 \mathrm{~km}(\mathrm{~d})($ unit: $\mathrm{m} / \mathrm{s})$.

weak or strong constraint on STMAS analysis have also been examined. We found that (1) the STMAS is superior to traditional 3D-Var for reconstructing the 3D typhoon structure, since the STMAS can retrieve multiscale information from observational network. (2) The radial velocity data provide additional useful information for the STMAS to reconstruct the detailed structure of 3D typhoon field. (3) Compared to a weak constraint, the strong constraint STMAS can produce better analyses on both horizontal and vertical velocities of the $3 \mathrm{D}$ typhoon structure.
This study gives us promising results. Challenges still remain when $3 \mathrm{D}$ radar radial velocity data are assimilated for the reconstruction and initialization of real typhoon structures in the future. First, given the fact that the real atmosphere is compressive, the wind vector may not satisfy the nondiffusivity continuity equation used in this study. Therefore, a full continuity equation should be used to make a more general strong constraint in future study. Second, the model error has not been taken into account in this study. The influence of model errors on typhoon reconstruction 


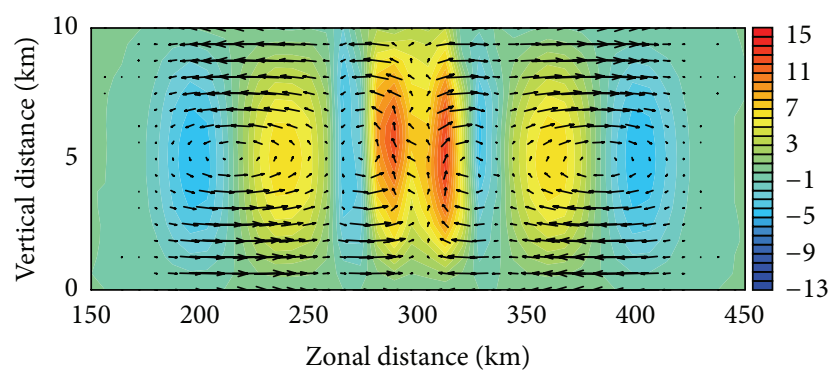

(a)

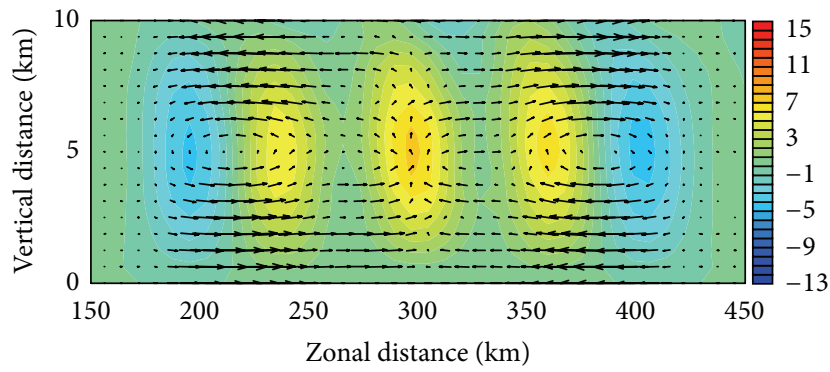

(c)

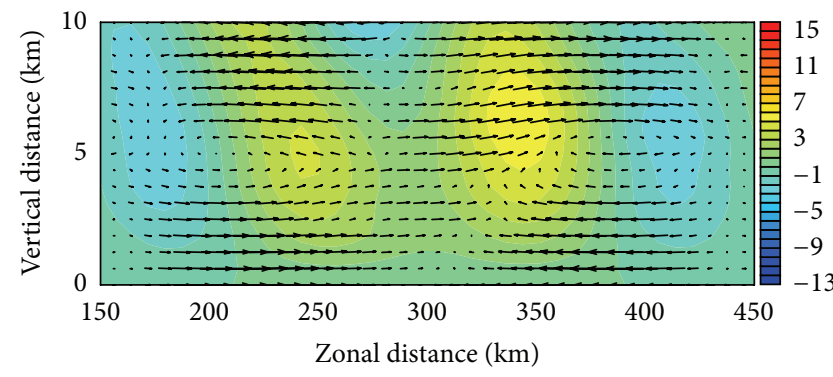

(b)

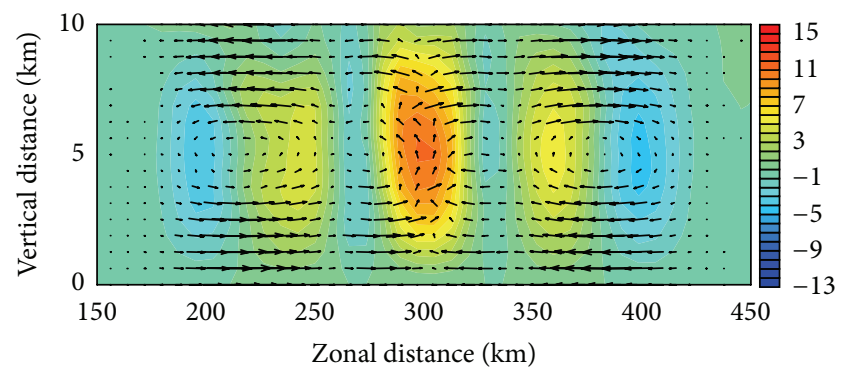

(d)

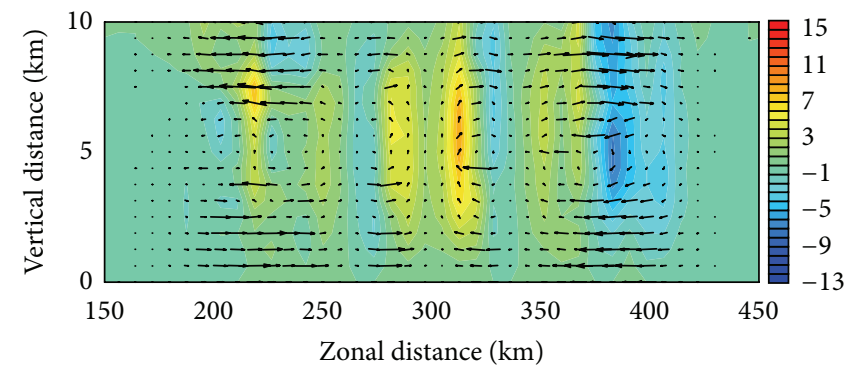

(e)

FIGURE 6: Vertical velocity section (color shade) and wind vector in this section across the typhoon center. (a) STMAS; (b) tradition 3D-Var with $L_{x}=50 \mathrm{~km}, L_{y}=50 \mathrm{~km}$, and $L_{z}=5 \mathrm{~km}$; (c) tradition $3 \mathrm{D}$-Var with $L_{x}=25 \mathrm{~km}, L_{y}=25 \mathrm{~km}$, and $L_{z}=2.5 \mathrm{~km}$; (d) tradition $3 \mathrm{D}$-Var with $L_{x}=12.5 \mathrm{~km}, L_{y}=12.5 \mathrm{~km}$, and $L_{z}=1.25 \mathrm{~km}$; (e) tradition $3 \mathrm{D}-\operatorname{Var}$ with $L_{x}=6.25 \mathrm{~km}, L_{y}=6.25 \mathrm{~km}$, and $L_{z}=0.625 \mathrm{~km}($ unit: $\mathrm{m} / \mathrm{s})$.

and initialization has to be addressed and how to deal with model errors could be an important research topic in the follow-up studies.

\section{Competing Interests}

The authors declare that there are no competing interests regarding the publication of this paper.

\section{Acknowledgments}

This research was jointly supported by grants of National Basic Research Program (2013CB430304), National Natural Science Foundation (41376013, 41376015, 41306006, 41541041, and 41506039), National High-Tech R\&D Program (2013AA09A505), and National Programme on Global Change and Air-Sea Interaction (GASI-01-01-12 and GASIIPOVAI-04) of China.

\section{References}

[1] J. Sun and N. A. Crook, "Real-time low-level wind and temperature analysis using single WSR-88D data," Weather and Forecasting, vol. 16, no. 1, pp. 117-132, 2001.

[2] S. S. Weygandt, A. Shapiro, and K. K. Droegemeier, "Retrieval of model initial fields from single-Doppler observations of a supercell thunderstorm. Part I: single-Doppler velocity retrieval," Monthly Weather Review, vol. 130, no. 3, pp. 433-453, 2002.

[3] S. S. Weygandt, A. Shapiro, and K. K. Droegemeier, "Retrieval of model initial fields from single-Doppler observations of a supercell thunderstorm. Part II: thermodynamic retrieval and numerical prediction," Monthly Weather Review, vol. 130, no. 3, pp. 454-476, 2002.

[4] Y.-C. Liou, J.-L. Chiou, W.-H. Chen, and H.-Y. Yu, "Improving the model convective storm quantitative precipitation nowcasting by assimilating state variables retrieved from multipleDoppler radar observations," Monthly Weather Review, vol. 142, no. 11, pp. 4017-4035, 2014. 
[5] J. Sun and N. A. Crook, "Dynamical and microphysical retrieval from Doppler radar observations using a cloud model and its adjoint-part I: model development and simulated data experiments," Journal of the Atmospheric Sciences, vol. 54, no. 12, pp. 1642-1661, 1997.

[6] J. Sun and N. A. Crook, "Dynamical and microphysical retrieval from Doppler radar observations using a cloud model and its adjoint. Part II: retrieval experiments of an observed Florida convective storm," Journal of the Atmospheric Sciences, vol. 55, no. 5, pp. 835-852, 1998.

[7] J. Gao, M. Xue, A. Shapiro, and K. K. Droegemeier, "A variational method for the analysis of three-dimensional wind fields from two Doppler radars," Monthly Weather Review, vol. 127, no. 9, pp. 2128-2142, 1999.

[8] M. Lindskog, H. Järvinen, and D. B. Michelson, "Development of radar wind data assimilation for the HIRLAM 3DVar," HIRLAM Technical Report 52, 2002.

[9] J.-D. Gao, M. Xue, K. Brewster, and K. K. Droegemeier, "A threedimensional variational data analysis method with recursive filter for Doppler radars," Journal of Atmospheric and Oceanic Technology, vol. 21, no. 3, pp. 457-469, 2004.

[10] Q. Xiao, Y.-H. Kuo, J. Sun et al., "Assimilation of Doppler radar observations with a regional 3DVAR system: impact of Doppler velocities on forecasts of a heavy rainfall case," Journal of Applied Meteorology, vol. 44, no. 6, pp. 768-788, 2005.

[11] J. Gao, M. Xue, S.-Y. Lee, A. Shapiro, Q. Xu, and K. K. Droegemeier, "A three-dimensional variational single-Doppler velocity retrieval method with simple conservation equation constraint," Meteorology and Atmospheric Physics, vol. 94, no. 1-4, pp. 11-26, 2006.

[12] M. Hu, M. Xue, and K. Brewster, "3DVAR and cloud analysis with WSR-88D level-II data for the prediction of the Fort Worth, Texas, tornadic thunderstorms. Part I: cloud analysis and its impact," Monthly Weather Review, vol. 134, no. 2, pp. 675-698, 2006.

[13] M. Hu, M. Xue, J. Gao, and K. Brewster, "3DVAR and cloud analysis with WSR-88D level-II data for the prediction of the Fort Worth, Texas, tornadic thunderstorms-part II: impact of radial velocity analysis via 3DVAR," Monthly Weather Review, vol. 134, no. 2, pp. 699-721, 2006.

[14] Q. Xiao and J. Sun, "Multiple-radar data assimilation and shortrange quantitative precipitation forecasting of a squall line observed during IHOP_2002," Monthly Weather Review, vol. 135, no. 10, pp. 3381-3404, 2007.

[15] S. Sugimoto, N. A. Crook, J. Sun, Q. Xiao, and D. Barker, “Assimilation of Doppler radar data with WRF 3DVAR: evaluation of its potential benefits to quantitative precipitation forecasting through Observing System Simulation Experiments," Monthly Weather Review, vol. 137, pp. 4011-4029, 2009.

[16] F. Q. Zhang, Y. Weng, J. A. Sippel, Z. Meng, and C. H. Bishop, "Cloud-resolving hurricane initialization and prediction through assimilation of doppler radar observations with an ensemble Kalman filter," Monthly Weather Review, vol. 137, no. 7, pp. 2105-2125, 2009.

[17] K. Zhao and M. Xue, "Assimilation of coastal Doppler radar data with the ARPS 3DVAR and cloud analysis for the prediction of Hurricane Ike (2008)," Geophysical Research Letters, vol. 36, no. 12, Article ID L12803, 2009.

[18] A. D. Schenkman, M. Xue, A. Shapiro, K. Brewster, and J. Gao, "The analysis and prediction of the 8-9 May 2007 Oklahoma tornadic mesoscale convective system by assimilating WSR88D and CASA radar data using 3DVAR," Monthly Weather Review, vol. 139, no. 1, pp. 224-246, 2011.
[19] Y. Z. Li, X. G. Wang, and M. Xue, "Assimilation of radar radial velocity data with the wrf hybrid ensemble-3DVAR system for the prediction of hurricane ike (2008)," Monthly Weather Review, vol. 140, no. 11, pp. 3507-3524, 2012.

[20] N. Du, M. Xue, K. Zhao, and J. Min, "Impact of assimilating airborne Doppler radar velocity data using the ARPS 3DVAR on the analysis and prediction of Hurricane Ike (2008)," Journal of Geophysical Research Atmospheres, vol. 117, no. 17, Article ID D18113, 2012.

[21] S. Abhilash, A. K. Sahai, K. Mohankumar, J. P. George, and S. Das, "Assimilation of Doppler weather radar radial velocity and reflectivity observations in WRF-3DVAR system for shortrange forecasting of convective storms," Pure and Applied Geophysics, vol. 169, no. 11, pp. 2047-2070, 2012.

[22] J. Dong and M. Xue, "Assimilation of radial velocity and reflectivity data from coastal WSR-88D radars using an ensemble Kalman filter for the analysis and forecast of landfalling hurricane Ike (2008)," Quarterly Journal of the Royal Meteorological Society, vol. 139, no. 671, pp. 467-487, 2013.

[23] L. Zhu, Q. Wan, X. Shen et al., "Prediction and predictability of high-impact Western Pacific landfalling Tropical Cyclone Vicente (2012) through convection-permitting ensemble assimilation of doppler radar velocity," Monthly Weather Review, vol. 144, no. 1, pp. 21-43, 2016.

[24] F. Shen, J. Min, and D. Xu, "Assimilation of radar radial velocity data with the WRF Hybrid ETKF-3DVAR system for the prediction of Hurricane Ike (2008)," Atmospheric Research, vol. 169, pp. 127-138, 2016.

[25] Y. F. Xie, S. E. Koch, J. A. McGinley, S. Albers, and N. Wang, "A sequential variational analysis approach for mesoscale data assimilation," in Proceedings of the 21st Conference on Weather Analysis and Forecasting/17th Conference on Numerical Weather Prediction, 15B.7, American Meteorological Society, Washington, DC, USA, 2005, http://ams.confex.com/ams/pdfpapers/ 93468.pdf.

[26] Y. Xie, S. Koch, J. McGinley et al., "A space-time multiscale analysis system: a sequential variational analysis approach," Monthly Weather Review, vol. 139, no. 4, pp. 1224-1240, 2011.

[27] Z. J. He, Y. F. Xie, W. Li et al., "Application of the sequential three-dimensional variational method to assimilating SST in a global ocean model," Journal of Atmospheric and Oceanic Technology, vol. 25, no. 6, pp. 1018-1033, 2008.

[28] W. Li, Y. Xie, Z. He et al., "Application of the multigrid data assimilation scheme to the China seas' temperature forecast," Journal of Atmospheric and Oceanic Technology, vol. 25, no. 11, pp. 2106-2116, 2008.

[29] W. Li, Y. F. Xie, S.-M. Deng, and Q. Wang, "Application of the multigrid method to the two-dimensional Doppler radar radial velocity data assimilation," Journal of Atmospheric and Oceanic Technology, vol. 27, no. 2, pp. 319-332, 2010.

[30] W. Li, Y. F. Xie, and G. J. Han, "A theoretical study on the multi-grid data assimilation scheme using a simple bilinear interpolation scheme," Acta Oceanologica Sinica, vol. 32, no. 3, pp. 80-87, 2013.

[31] R. Fletcher, Practical Methods of Optimization, John Wiley \& Sons, Chichester, UK, 2nd edition, 1987.

[32] R. H. Byrd, P. Lu, J. Nocedal, and C. Y. Zhu, "A limited memory algorithm for bound constrained optimization," SIAM Journal on Scientific Computing, vol. 16, no. 5, pp. 1190-1208, 1995.

[33] J. Derber and A. Rosati, "A global oceanic data assimilation system," Journal of Physical Oceanography, vol. 19, no. 9, pp. 1333-1347, 1989. 

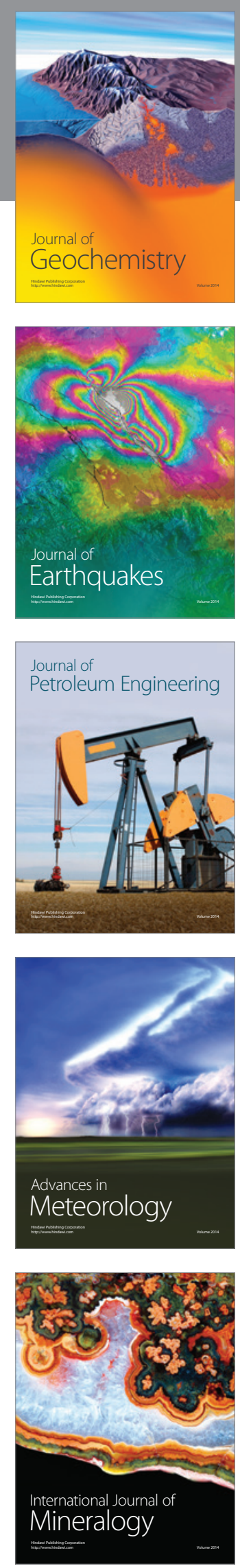
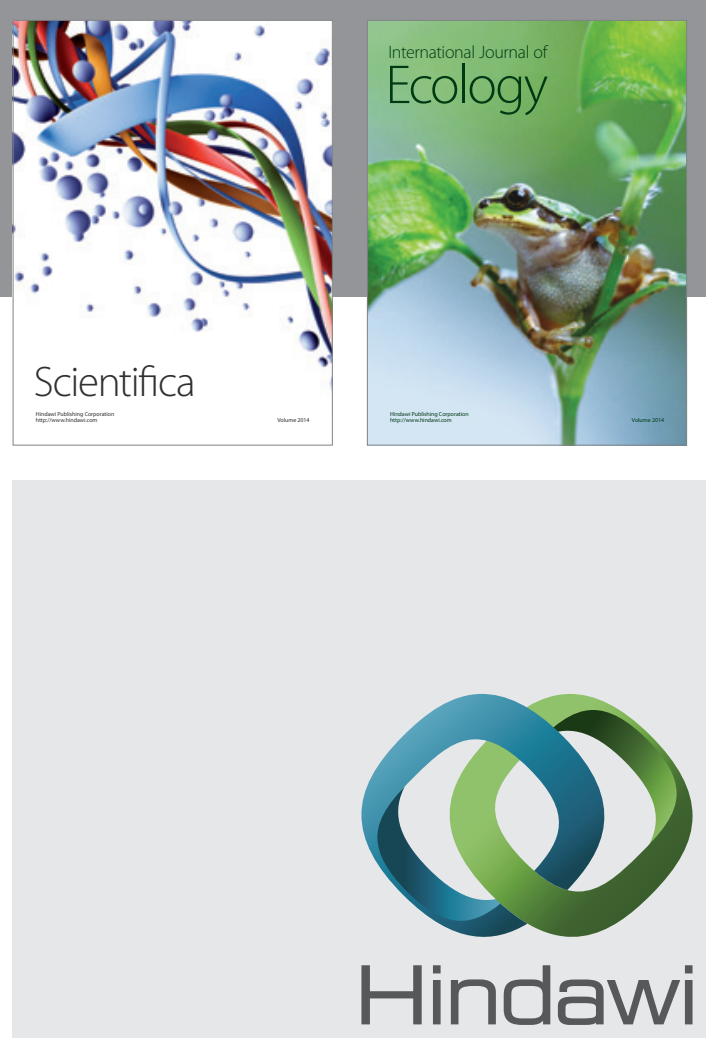

Submit your manuscripts at

http://www.hindawi.com
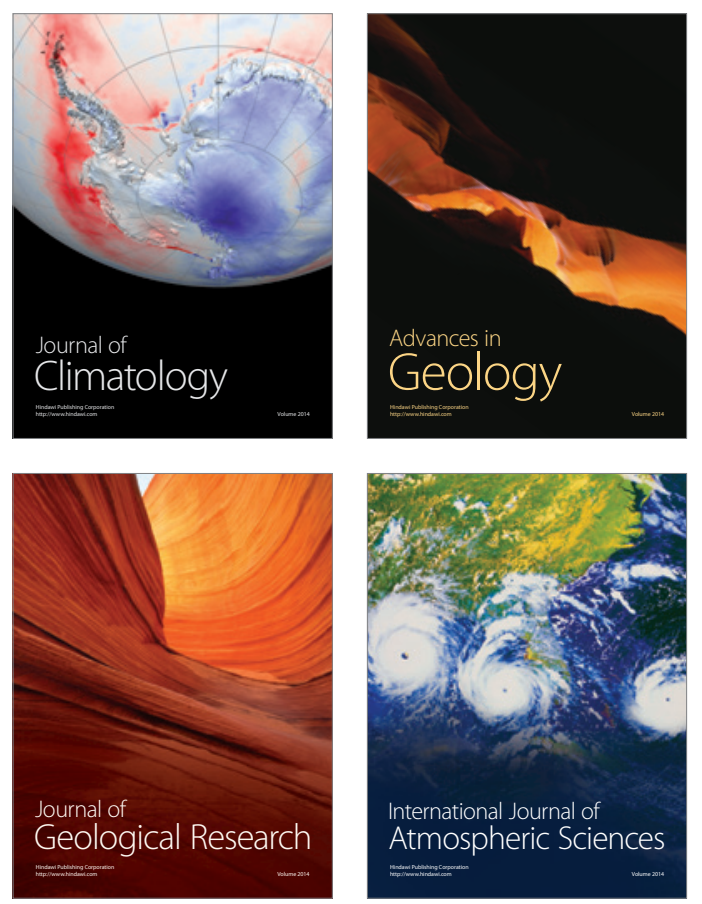

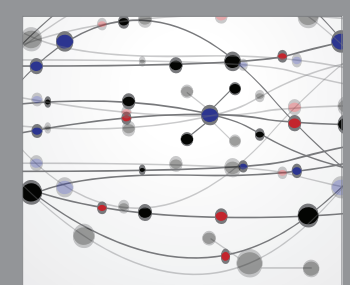

The Scientific

\section{World Journal}
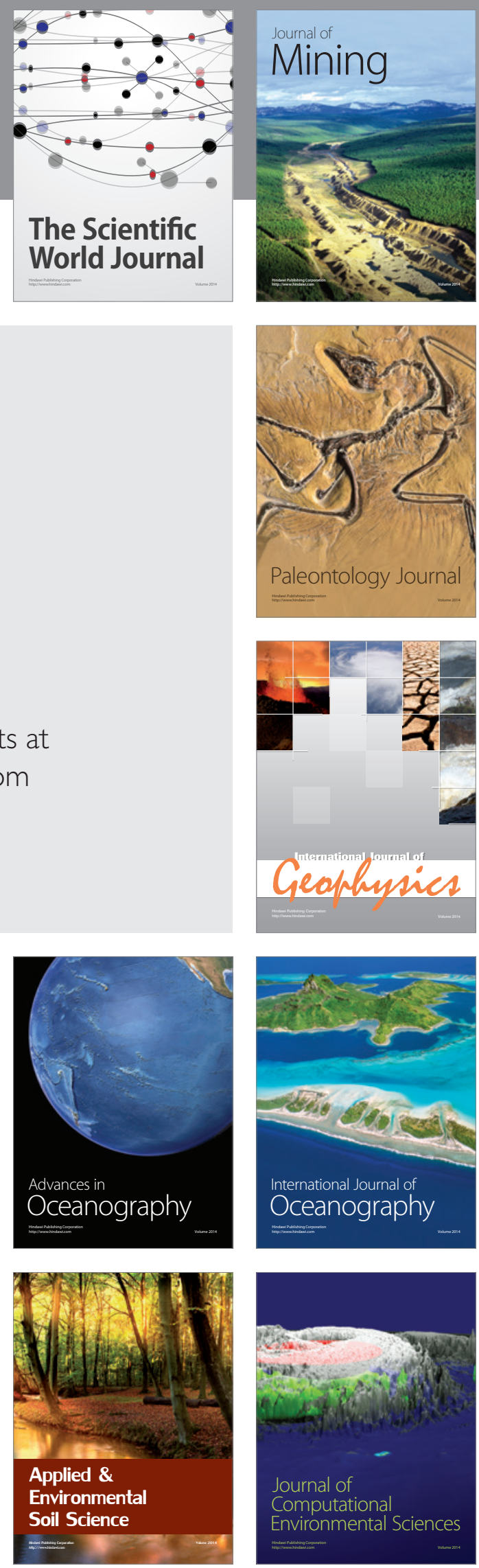